\title{
A model for anomalous moisture diffusion through a polymer-clay nanocomposite
}

\author{
A.D. Drozdov*, J. deC. Christiansen \\ Department of Production \\ Aalborg University, Fibigerstraede 16 \\ DK-9220 Aalborg, Denmark \\ and \\ R.K. Gupta, A.P. Shah ${ }^{\dagger}$ \\ Department of Chemical Engineering and Constructed Facilities Center \\ West Virginia University, P.O. Box 6102 \\ Morgantown, WV 26506, USA
}

\begin{abstract}
Experimental data are reported on moisture diffusion and the elastoplastic response of an intercalated nanocomposite with vinyl ester resin matrix and montmorillonite clay filler at room temperature. Observations in diffusion tests show that water transport in the neat resin is Fickian, whereas it becomes anomalous (non-Fickian) with the growth of the clay content. This transition is attributed to immobilization of penetrant molecules on the surfaces of hydrophilic clay layers. Observations in uniaxial tensile tests demonstrate that the response of vinyl ester resin is strongly elastoplastic, whereas an increase in the clay content results in a severe decrease of plastic strains observed as a noticeable reduction of curvatures of the stress-strain diagrams. This is explained by slowing down of molecular mobility in the host matrix driven by confinement of chains in galleries between platelets. Constitutive equations are developed for the anomalous moisture diffusion through and the elastoplastic behavior of a nanocomposite. Adjustable parameters in these relations are found by fitting the experimental data. Fair agreement is demonstrated between the observations and the results of numerical simulation. A striking similarity is revealed between changes in diffusivity, ultimate water uptake and the rate of plastic flow with an increase in the clay content.
\end{abstract}

Key-words: Anomalous diffusion, Elastoplasticity, Nanocomposite

\footnotetext{
${ }^{*}$ Corresponding author, fax: +45 9815 3030, E-mail: drozdov@iprod.auc.dk

${ }^{\dagger}$ Present address: Triton Systems Inc., 200 Turnpike Road, Chelmsford, MA 01824, USA
} 


\section{Introduction}

This paper is concerned with modelling moisture diffusion through a hybrid composite consisting of a polymer matrix filled with clay nanoparticles. Permeation of small molecules through polymer-clay nanocomposites has attracted substantial attention in the past decade, see, e.g., [1, 2, 3, 4, 5, 6, 7, 8, 9, 10, 11, 12, to mention a few. This may be explained by a strongly increasing interest to development and characterization of nanocomposites whose physical properties demonstrate a noticeably improvement compared to those of pristine polymers at rather low (conventionally, less than 5\%) filler concentrations.

In the previous works, barrier properties of polymer-clay nanocomposites were measured by the permeability cup method [1, 4, 5, 6, 10] and the microgravimetric technique [9, 11, 12]. The former approach characterizes the steady-state mass transport only. In the latter case, the microgravimetric analysis focused only on (i) the initial parts of the mass gain curves (which provided estimates of diffusivity for the Fickian diffusion) and (ii) the equilibrium water uptakes. This implies that the kinetics of moisture diffusion in polymer-clay nanocomposites have not yet been investigated in detail.

Changes in diffusivity and permeability of nanocomposites with clay content are conventionally explained within the concept of tortuous paths [13. According to this approach, the path that a small molecule of a penetrant must travel in a polymeric matrix substantially increases in the presence of intercalated and/or exfoliated clay layers (that possess aspect ratios of order of $10^{3}$ ). This results in a noticeable decrease in the coefficient of diffusion (estimated as an average value of the square of the end-to-end distance passed per unit time). According to the Nielsen equation [13], the tortuosity factor (the ratio of diffusivity of a composite to that of the pristine polymer) is inversely proportional to a linear function of the volume fraction of filler. Among other phenomenological relations, it is worth noting the Cussler formula [14] (the tortuosity factor is inversely proportional to a linear function of the square of the volume fraction of filler), the Barrer formula [6] (the tortuosity factor is proportional to the square of the volume fraction of polymer), and the power-law equation [15] (the tortuosity factor is proportional to a fractional power of the volume fraction of matrix). The Nielsen formula was recently used to fit experimental data for polyimide-clay [1, 5] and polyester-clay [8] nanocomposites. The Barrer equation was employed to match observations for Nylon 11 filled with nanosize silica [6]. In all cases, the quality of approximation was far from being excellent.

To improve the accuracy of fitting, Fredrickson and Bicerano [16] and Bharadwaj 17] proposed phenomenological relations that account for the orientation of platelets, their shape, and the state of delamination. This (merely geometrical) approach may

reduce deviations between experimental data and results of numerical simulation (due to an increase in the number of adjustable parameters), but it cannot overcome the main shortcoming of the original concept: the tortuous path theory is grounded on the assumption that the presence of nanoparticles does not affect the diffusivity of a polymer matrix 16.

This postulate appears to be rather questionable, because observations show that mass transport in polymers is intimately connected with their viscoelastic properties [18]. Experimental data in oscillation tests with small strains reveal an increase in the storage 
modulus and a decrease in the loss modulus with filler content, $\nu$, for nanocomposites with Nylon 6 [19], Nylon 11 [6], Nylon 1012 [12] and polyester [8] matrices. The reduction of the loss tangent (that reflects changes in the viscoelastic response) is accompanied by a decay in viscoplastic properties revealed as formation of a pronounced yield point for polypropylene-clay nanocomposites and transition from ductile to brittle fraction in Nylon 6-clay nanocomposites with the growth of the filler fraction [19]. These observations demonstrate that mobility of macromolecules in a polymeric matrix slows down with the clay content. Adopting the concept of "chain immobilization factor," see [15] and the references therein, one can conclude that this deceleration of molecular mobility should be accompanied by a decrease in diffusivity of small molecules, which is not accounted for by the concept of tortuous paths.

The objective of this study is three-fold:

1. To report experimental data in moisture diffusion tests and in uniaxial tensile tests on vinyl ester resin-montmorillonite clay nanocomposite with various contents of filler.

2. To derive constitutive equations for water sorption and stress-strain relations for uniaxial deformation of a nanocomposite and to find adjustable parameters in the governing equations by fitting the observations.

3. To demonstrate that the clay fraction affects the coefficient of diffusion and the constants responsible for the viscoplastic behavior in a similar way.

The choice of vinyl ester resin for the experimental analysis is explained by numerous applications of this thermosetting polymer as a matrix for glass-reinforced polymer composites employed in construction and repair of civil structures [20].

Montmorillonite (MMT) is an inorganic clay conventionally used for preparation of hybrid nanocomposites. It possesses a layered structure constructed of two tetrahedral sheets of silica surrounding an octahedral sheet of alumina or magnesia. The layers (with thickness of $1 \mathrm{~nm}$ ) are stacked by weak dipole forces, while the galleries between the layers are occupied by metal cations.

To evaluate the effect of clay content on the mobility of macromolecules in the matrix, we concentrate of water sorption tests and uniaxial tensile tests. Our experimental data (Figures 1 to 5) demonstrate that moisture diffusion in the neat vinyl ester resin is Fickian. With an increase in the clay concentration, the Fickian diffusion is transformed into an anomalous transport of the penetrant molecules. Anomalous diffusion of water in polymers and polymer composites has been recently studied in [21, 22, 23, 24, 25].

We suppose that this anomalous moisture uptake in a nanocomposite filled with MMT particles may be explained by immobilization of water molecules on the surfaces of hydrophilic clay layers. A model for a two-stage diffusion with immobilization of penetrant molecules was proposed by Carter and Kibler [26] and Gurtin and Yatomi [27]. Unlike these works, we assume that bounded molecules cannot leave the sites where they were immobilized, which allows the number of adjustable parameters to be substantially reduced. The diffusion process is described by differential equations with three material constants that are found by matching the water uptake curves plotted in Figures 1 to 5 . 
Our observations in tensile tests (Figures 6 to 10) reveal that the stress-strain curve for a pristine polymer substantially differs from a straight line, which means that the mechanical response of the neat vinyl ester resin is strongly elastoplastic. With an increase in the clay content, the stress-strain diagrams become "less curved," which is associated with a decay in the material plasticity. Similar observations were recently reported for nanocomposites with Nylon 6, polypropylene [19], and poly $(\varepsilon$-caprolactone) [9] matrices filled with MMT clay.

To describe the elastoplastic behavior, we treat a nanocomposite as an equivalent network of chains bridged by junctions (entanglements, physical and chemical cross-links, and clay platelets). The elastic response is attributed to elongations of strands in the network, whereas the plastic response is attributed to sliding of junctions with respect to their reference positions in the medium (the non-affine deformation of the network). The decrease in the curvatures of the stress-strain diagrams with the clay content is ascribed to a decay in mobility of junctions whose motion is restricted by the presence of nanoparticles. A model is developed to describe constrains imposed by the clay platelets on the mobility of chains in the host matrix. The constitutive equations are determined by three adjustable parameters with a transparent physical meaning. These parameters are found by matching the stress-strain curves in uniaxial tensile tests with small strains.

Unlike the previous study [28], where observations were reported for a nanocomposite filled with MMT clay treated with dimethyl benzyl hydrogenated tallow quaternary ammonium chloride (which implied that the clay layers were not chemicaly bonded to chains in the host matrix), the present work focuses on transport of water molecules through a nanocomposite filled with MMT clay modified with vinyl benzyl ammonium chloride (the latter ensures that some macromolecules are chemically bonded to the clay platelets).

The exposition is organized as follows. Observations in moisture diffusion tests and uniaxial tensile tests are reported in Section 2. A model for water diffusion through a nanocomposite is derived in Section 3 and its material constants are found in Section 4. Constitutive equations for the elastoplastic response of a nanocomposite are developed in Section 5. Adjustable parameters in the stress-strain relations are determined in Section 6. A brief discussion of our findings is presented in Section 7. Some concluding remarks are formulated in Section 8.

\section{Experimental procedure}

\subsection{Preparation of samples}

The polymer used was DERAKANE 411-350 epoxy vinyl ester resin (Dow Chemical Co.) containing 45 wt.- $\%$ of dissolved styrene. To cure the resin at room temperature, it was mixed with 0.5 wt.- $\%$ of $6 \%$ cobalt naphthenate catalyst (Sigma Aldrich Co.). Additionally, $0.05 \%$ of $99 \%$ N,N dimethyl aniline (Lancaster Synthesis, Pelham, NH, USA) was used as an accelerator, and $1.5 \%$ of methyl ethyl ketone peroxide with $9 \%$ of active oxygen (Sigma Aldrich Co.) as an initiator.

A sodium montmorillonite clay Cloisite NA (Southern Clay Products Inc., Gonzales, TX, USA) was treated with vinyl benzyl ammonium chloride (Sigma Aldrich Co.). $30 \mathrm{~g}$ of Cloisite NA was dispersed in $800 \mathrm{ml}$ of distilled water. Separately, $6.35 \mathrm{~g}$ of vinyl benzyl 
ammonium chloride (that corresponds to $100 \mathrm{meq} / 100 \mathrm{~g}$ of clay) was dissolved in $80 \mathrm{ml}$ of distilled water. After stirring of the clay dispersion with a mechanical stirrer for $5 \mathrm{~h}$, the vinyl benzyl ammonium chloride solution was added drop by drop in the dispersion. The mixture was stirred for another $3 \mathrm{~h}$, and the organo-MMT clay was filtered using a vacuum filter, dried in an oven at $100{ }^{\circ} \mathrm{C}$, and ground in a ball mill.

Neat resin coupons were cast by pouring the reaction mixture into Teflon molds with the dimensions $50 \times 12.5 \mathrm{~mm}$ and the thickness ranging from 0.2 to $0.6 \mathrm{~mm}$. The organically treated clay was added to the liquid resin and manually stirred. The mixture was then degassed in a vacuum oven to remove air bubbles. Afterwards, the catalyst, initiator and accelerator were added. The mixture was allowed to cure at room temperature for 24 hours, and it was subsequently post-cured in an oven for 3 hours at $90{ }^{\circ} \mathrm{C}$.

TEM (transmission electron microscopy) micrographs of samples show that an intercalated nanocomposite is produced [29]. It is worth noting, however, that the thickness of interlayers between platelets does not increase substantially, which may be explained by cross-linking between unsaturated sites on the surfaces of clay layers and vinyl groups of chains in the host matrix 29, 30].

\subsection{Diffusion tests}

Diffusion tests were performed by immersing samples with rectangular cross-section in distilled water at room temperature $\left(T=25^{\circ} \mathrm{C}\right)$. The samples had a dry mass ranging from 120 to $400 \mathrm{mg}$. They were stored in a controlled humidity chamber, and contained $0.05 \pm 0.005$ wt.- $\%$ of water at the beginning of diffusion tests. The samples were periodically removed, blotted dry with a lint-free tissue, weighed and re-immersed into water. A typical experiment lasted ten days, and, on the first day, readings were taken as frequently as every $15 \mathrm{~min}$. The balance used had an accuracy of $1 \mathrm{mg}$, and 3 to 5 replicate runs were carried out for a given set of condition.

Experimental data are presented in the form of a relative mass gain, $\Phi$, as a function of a reduced time, $\bar{t}$. The reduced time, $\bar{t}$, is given by

$$
\bar{t}=\frac{t}{4 l^{2}},
$$

where $t$ is time elapsed from the beginning of a test, and $2 l$ is thickness of a sample. The relative moisture uptake, $\Phi$, reads

$$
\Phi(\bar{t})=\frac{R(\bar{t})}{R(\infty)},
$$

where $R(\bar{t})$ is the mass gain at the reduced time $\bar{t}$ and $R(\infty)$ is the maximal mass gain.

Typical moisture uptake curves are plotted in Figures 1 to 5. These figures demonstrate that diffusion of water molecules in the neat resin is Fickian, but it becomes strongly anomalous with an increase in the clay content.

\subsection{Mechanical tests}

Dumbbell specimens for mechanical tests were prepared in accordance with ASTM D638 specification. Tensile tests were performed at ambient temperature by using a $100 \mathrm{kN}$ 
Instron machine model 8501 with a cross-head speed of $0.254 \mathrm{~mm} / \mathrm{min}$. This strain rate ensures practically isothermal loading conditions. The strain was measured independently using a strain gauge affixed to the mid-point of a specimen. The tensile force was measured by a standard load cell. The engineering stress, $\sigma$, was determined as the ratio of the tensile force to the cross-sectional area of a specimen in the stress-free state. For any clay concentration, $\nu$, four specimens were tested. Typical stress-strain diagrams are presented in Figures 6 to 10. These figures show that the mechanical response of the neat resin is strongly nonlinear, but the nonlinearity of the stress-strain curves is reduced with an increase in the clay content.

DSC (differential scanning calorimetry) measurements show that the glass transition temperature of the neat vinyl ester resin is about $98^{\circ} \mathrm{C}$. It increases with the clay content reaching $112{ }^{\circ} \mathrm{C}$ at $\nu=1.0$ wt.- $\%$, and remains practically constant at higher concentrations of filler [29]. At room temperature, the nanocomposite is far below its glass transition point, which implies that we can disregard its viscoelastic response and attribute the nonlinearity of the stress-strain curves to the elastoplastic behavior.

\section{A model for moisture diffusion}

A sample is treated as a rectilinear plate with thickness $2 l$. We introduce Cartesian coordinates $\{x, y, z\}$, where the axis $x$ is perpendicular to the middle plane of the plate, and the axes $y$ and $z$ are located in the middle plane. Length and width of the plate substantially exceed its thickness, which implies that the moisture concentration depends on the only coordinate $x$.

After the plate is immersed into water, three processes occur in the nanocomposite:

1. sorption of water molecules to the sample faces from the surrounding,

2. diffusion of the penetrant into the plate,

3. adsorption of water molecules on the hydrophilic surfaces of clay layers, where these molecules become immobilized.

It is conventionally accepted that the rate of sorption in glassy polymers noticeably exceeds the rate of diffusion [24], which implies that the sorption equilibrium is rapidly established. The equilibrium condition reads

$$
\left.n(t, x)\right|_{x= \pm l}=n^{\circ}
$$

where $n$ is the moisture concentration at time $t$ at point $x$ [the number of water molecules in the polymeric matrix occupying a volume with the unit area in the plane $(y, z)$ and the thickness $d x$ reads $n(t, x) d x]$, and $n^{\circ}$ is the equilibrium moisture concentration in the matrix on the faces of a sample.

Diffusion of a penetrant through a matrix is described by the mass conservation law

$$
\frac{\partial n}{\partial t}=\frac{\partial J}{\partial x}-\frac{\partial n_{1}}{\partial t}
$$


where $n_{1}(t, x)$ denotes the concentration of water molecules immobilized at the surfaces of clay layers, and the mass flux, $J(t, x)$, is given by the Fick formula

$$
J=D \frac{\partial n}{\partial x}
$$

The constant $D$ in Eq. (5) stands for the coefficient of diffusion through the polymer (which means that only the mass transport through the host matrix is taken into account). It is assumed, however, that $D$ depends on the clay content: the presence of clay platelets in the polymeric matrix leads to a decrease in the chain mobility that provides a driving force for diffusion.

Adsorption of water molecules on the hydrophilic surfaces of clay layers is governed by the first-order differential equation

$$
\frac{\partial n_{1}}{\partial t}=k n\left(n_{1}^{\circ}-n_{1}\right)
$$

Equation (6) means that the rate of immobilization of small molecules is proportional to (i) the penetrant concentration in the matrix, $n$, and (ii) the number of "unoccupied sites" on the surfaces of clay platelets, $n_{1}^{\circ}-n_{1}$, where $n_{1}^{\circ}$ stands for the total number of sites where water molecules are bounded. The rate of adsorption, $k$, and the maximal concentration of unoccupied sites, $n_{1}^{\circ}$, are assumed to depent on the clay content, because the presence of nanoparticles affects mobility of chains in the polymeric matrix, and, as a consequence, its chemical potential.

The following initial conditions are accepted:

$$
\left.n(t, x)\right|_{t=0}=0,\left.\quad n_{1}(t, x)\right|_{t=0}=0,
$$

which mean that the moisture content in a sample before testing is disregarded.

The water mass gain (per unit mass of the sample) is given by

$$
R(t)=\frac{\kappa}{2 l \rho} \int_{-l}^{l}\left[n(t, x)+n_{1}(t, x)\right] d x
$$

where $\kappa$ is the average mass of a penetrant molecule, $\rho$ is mass density of the polymeric matrix, and the functions $n$ and $n_{1}$ obey Eqs. (3) to (7).

Our aim now is to transform the governing equations. Introducing the dimensionless variables

$$
\bar{x}=\frac{x}{l}, \quad c=\frac{n}{n^{\circ}}, \quad c_{1}=\frac{n_{1}}{n^{\circ}}
$$

and combining Eqs. (1), (4) and (5), we arrive at the mass flux equation

$$
\frac{\partial c}{\partial \bar{t}}=\bar{D} \frac{\partial^{2} c}{\partial \bar{x}^{2}}-\frac{\partial c_{1}}{\partial \bar{t}}
$$

where

$$
\bar{D}=4 D
$$

In the new notation, Eq. (6) reads

$$
\frac{\partial c_{1}}{\partial \bar{t}}=K c\left(C-c_{1}\right)
$$


where

$$
K=4 k l^{2} n^{\circ}, \quad C=\frac{n_{1}^{\circ}}{n^{\circ}} .
$$

It follows from Eq. (7) that the initial conditions for Eqs. (9) and (11) are given by

$$
c(0, \bar{x})=0, \quad c_{1}(0, \bar{x})=0 .
$$

Bearing in mind that the functions $c(\bar{t}, \bar{x})$ and $c_{1}(\bar{t}, \bar{x})$ are even functions of $\bar{x}$, we find from Eq. (3) that

$$
\frac{\partial c}{\partial \bar{x}}(\bar{t}, 0)=0, \quad c(\bar{t}, 1)=1
$$

Equation (8) implies that

$$
R(\bar{t})=\frac{\kappa}{\rho} n^{\circ} \int_{0}^{1}\left[c(\bar{t}, \bar{x})+c_{1}(\bar{t}, \bar{x})\right] d \bar{x} .
$$

According to Eqs. (9) and (11), with an increase in time, $\bar{t}$, the functions $c$ and $c_{1}$ approach their steady-state values

$$
c(\infty, \bar{x})=1, \quad c_{1}(\infty, \bar{x})=C .
$$

Equations (15) and (16) result in the formula for the maximal moisture uptake,

$$
R(\infty)=\frac{\kappa}{\rho} n^{\circ}(1+C)
$$

The relative mass gain is found from Eqs. (2), (15) and (17),

$$
\Phi(\bar{t})=\frac{1}{1+C} \int_{0}^{1}\left[c(\bar{t}, \bar{x})+c_{1}(\bar{t}, \bar{x})\right] d \bar{x} .
$$

Equations (9), (11) and (18) with initial conditions (13) and boundary conditions (14) are determined by 3 adjustable parameters:

1. the reduced diffusivity $\bar{D}$,

2. the rate of moisture adsorption, $K$, on the hydrophilic surfaces of clay layers,

3. the reduced concentration of unoccupied sites, $C$, on the surfaces of clay platelets.

These quantities are found by fitting the experimental data in diffusion tests.

\section{Fitting observations in diffusion tests}

We begin with matching the experimental data for the neat vinyl resin depicted in Figure 1. In the absence of clay particles, one can set $C=0$. This equality together with Eqs. (11) and (13) implies that $c_{1}(t, x)=0$. Bearing in mind this formula, we find from Eq.

(9) that the function $c$ obeys the conventional diffusion equation

$$
\frac{\partial c}{\partial \bar{t}}=\bar{D} \frac{\partial^{2} c}{\partial \bar{x}^{2}}
$$


with initial condition (13) and boundary conditions (14). The initial-boundary problem (13), (14) and (19) is solved numerically by the finite-difference method with an explicit algorithm. We divide the interval $[0,1]$ into $M$ subintervals by points $\bar{x}_{m}=m \Delta x(m=$ $0,1, \ldots, M)$ with $\Delta x=1 / M$, introduce discrete time $\bar{t}_{n}=n \Delta t$, and replace Eqs. (13), (14) and (19) by their finite-difference approximation

$$
\begin{aligned}
c\left(0, \bar{x}_{m}\right)= & 0 \quad(m=0,1, \ldots, M-1), \quad c\left(0, \bar{x}_{M}\right)=1, \\
c\left(\bar{t}_{n+1}, \bar{x}_{m}\right)= & c\left(\bar{t}_{n}, \bar{x}_{m}\right)+\bar{D} \frac{\Delta t}{\Delta x^{2}}\left[c\left(\bar{t}_{n}, \bar{x}_{m+1}\right)-2 c\left(\bar{t}_{n}, \bar{x}_{m}\right)\right. \\
& \left.+c\left(\bar{t}_{n}, \bar{x}_{m-1}\right)\right] \quad(m=1, \ldots, M-1), \\
c\left(\bar{t}_{n+1}, \bar{x}_{0}\right)= & c\left(\bar{t}_{n+1}, \bar{x}_{1}\right), \quad c\left(\bar{t}_{n+1}, \bar{x}_{M}\right)=1 \quad(n=1,2, \ldots) .
\end{aligned}
$$

The relative mass gain is given by Eq. (18), where the integral is estimated by means of the Euler formula,

$$
\Phi\left(\bar{t}_{n}\right)=\Delta x \sum_{m=0}^{M-1} c\left(\bar{t}_{n}, \bar{x}_{m}\right) .
$$

The coefficient $\bar{D}$ is determined by the following procedure. First, we fix some interval $\left[D_{\min }, D_{\max }\right]$, where the "best-fit" value of $\bar{D}$ is supposed to be located, and divide this interval into $I$ sub-intervals by the points $\bar{D}_{i}=D_{\min }+i \Delta D(i=0,1, \ldots, I)$, where $\Delta D=\left(D_{\max }-D_{\min }\right) / I$. For any $\bar{D}_{i}$, Eq. (20) is solved numerically (with the steps $\Delta x=0.05$ and $\Delta t=0.005$ that guarantee the stability of the numerical algorithm), and the function $\Phi\left(\bar{t}_{n}\right)$ is given by Eq. (21). The "best-fit" value of $\bar{D}$ is found from the condition of minimum of the function

$$
F=\sum_{\bar{t}_{k}}\left[\Phi_{\exp }\left(\bar{t}_{k}\right)-\Phi_{\text {num }}\left(\bar{t}_{k}\right)\right]^{2},
$$

where the sum is calculated over all point, $\bar{t}_{k}$, depicted in Figure $1, \Phi_{\exp }\left(\bar{t}_{k}\right)$ is the relative mass gain measured in the test, and $\Phi_{\text {num }}\left(\bar{t}_{k}\right)$ is determined by Eq. (21). After the "bestfit" coefficient, $\bar{D}_{i}$, is found, this procedure is repeated for the new interval $\left[\bar{D}_{i-1}, \bar{D}_{i+1}\right]$ to ensure an acceptable accuracy of matching observations.

To show that the numerical results are not affected by our choice of the steps of integration, $\Delta x$ and $\Delta t$, we solve Eqs. (20) and (21) numerically with the "best-fit" parameter $\bar{D}$ and the new steps, $\Delta x=0.01$ and $\Delta t=0.0005$. No difference is visible between the curve plotted in Figure 1 and the curve obtained by numerical integration of Eqs. (20) and (21) with the decreased steps in time and the spatial coordinate.

The above procedure is repeated to match observations in the tests on 4 specimens with various thicknesses, and the average diffusivity (over 4 specimens) and its standard deviation are determined.

The same numerical algorithm is employed to fit experimental data on nanocomposites with various contents of clay. The difference in the treatment of observations consists in the following:

1. Eq. (20) is replaced by an appropriate finite-difference approximation of Eqs. (9) and (11), 
2. the "best-fit" values are simultaneously determined for 3 adjustable parameters, $\bar{D}$, $C$ and $K$.

Figures 1 to 5 demonstrate fair agreement between the experimental data and the results of numerical analysis.

It is worth noting that our algorithm for the approximation of observations differs from that employed in [9, 11, 29]. In the previous works, the coefficient of diffusion was determined by matching the initial parts of the mass gain diagrams (where the function $\Phi(\bar{t})$ is approximately linear), which did not allow immobilization of water molecules on the surfaces of clay layers to be accounted for.

The average values of $D$ [given $\bar{D}$, the diffusivity, $D$, is determined by Eq. (10)], $C$ and $K$ (over 4 tests) are plotted versus the clay content in Figures 11 to 13 (the vertical bars stand for the standard deviations). The dependence of diffusivity, $D$, on the clay content, $\nu$, is approximated by the phenomenological equation

$$
D=D_{0}+D_{1} \exp \left(-\frac{\nu}{\nu_{0}}\right),
$$

where $D_{0}, D_{1}$ and $\nu_{0}$ are adjustable parameters. The quantity $\nu_{0}$ is determined by the steepest-descent method to minimize deviations between the experimental data and their approximation by Eq. (22). The coefficients $D_{0}$ and $D_{1}$ are found by the least-squares algorithm.

The effect of clay content, $\nu$, on the steady concentration, $C$, of immobilized water molecules is predicted by the linear function

$$
C=C_{1} \nu
$$

where the coefficient $C_{1}$ is determined by the least-squares technique.

To describe the influence of clay content, $\nu$, on the dimensionless rate, $K$, of water adsorption on the clay platelets, we employ the phenomenological relation similar to Eq. $(22)$,

$$
\log K=K_{0}+K_{1} \exp \left(-\frac{\nu}{\nu_{0}}\right),
$$

where $\log =\log _{10}$. We use the same value, $\nu_{0}=0.55$, that was found by fitting observations for the coefficient of diffusion with the help of Eq. (22). The material parameters, $K_{0}$ and $K_{1}$, in Eq. (24) are determined by the least-squares method.

It follows from Eq. (17) that the maximal moisture uptake by the polymeric matrix, $R_{\mathrm{pol}}=\kappa n^{\circ} / \rho$, reads

$$
R_{\mathrm{pol}}=\frac{R(\infty)}{1+C} .
$$

Using measurements of the maximal mass gain, $R(\infty)$, and the values of $C$ determined by matching observations, we calculate $R_{\text {pol }}$ from Eq. (25). This quantity is plotted versus clay content, $\nu$, in Figure 14. By analogy with Eq. (22), we fit the experimental data by the equation

$$
R_{\mathrm{pol}}=R_{0}-R_{1} \exp \left(-\frac{\nu}{\nu_{0}}\right),
$$

where the coefficients $R_{0}$ and $R_{1}$ are found by the least-squares algorithm. 
Equations (12) and (17) imply that the maximal moisture uptake by the clay particles, $R_{\text {clay }}=\kappa n_{1}^{\circ} / \rho$, is given by

$$
R_{\text {clay }}=R(\infty)-R_{\text {pol }}
$$

We determine $R_{\text {clay }}$ from this equality and present this quantity as a function of clay content, $\nu$, in Figure 15. The experimental data are approximated by the linear function

$$
R_{\text {clay }}=R_{1} \nu
$$

where the constant $R_{1}$ is found by the least-squares technique.

Figures 11 to 15 demonstrate an acceptable agreement between the observations and their approximations by phenomenological relations (22) to (24), (26) and (27).

\section{A model for the elastoplastic response}

A nanocomposite is modelled as an equivalent network of chains bridged by junctions. Because the viscoelastic behavior of the nanocomposite far below the glass transition point is disregarded, the network is treated as permanent (which means that within the experimental time-scale, chains cannot separate from junctions as they are agitated by thermal fluctuations). The elastoplastic behavior of the network is treated as sliding of junctions with respect to their reference positions in the bulk medium.

For uniaxial deformation, sliding of junctions is determined by a plastic strain $\epsilon_{\mathrm{p}}$. Adopting the conventional hypothesis that the macro-strain, $\epsilon$, is transmitted to all chains in the network by surrounding macromolecules, we find that

$$
\epsilon=\epsilon_{\mathrm{e}}+\epsilon_{\mathrm{p}}
$$

where $\epsilon_{\mathrm{e}}$ is an elastic strain. We adopt the mean-field approach, according to which $\epsilon_{\mathrm{e}}$ and $\epsilon_{\mathrm{p}}$ are treated as average elastic and plastic strains per chain.

The rate of changes in the strain, $\epsilon_{\mathrm{p}}$, with time, $t$, is assumed to be proportional to the rate of changes in the macro-strain $\epsilon$,

$$
\frac{d \epsilon_{\mathrm{p}}}{d t}(t)=\varphi\left(\epsilon_{\mathrm{e}}(t)\right) \frac{d \epsilon}{d t}(t)
$$

where the coefficient of proportionality, $\varphi$, is a function of the elastic strain $\epsilon_{\mathrm{e}}$. The function $\varphi\left(\epsilon_{\mathrm{e}}\right)$ vanishes at the zero elastic strain, $\varphi(0)=0$, monotonically increases with $\epsilon_{\mathrm{e}}$, and approaches some constant $a \in(0,1)$ at relatively large elastic strains. The constant

$$
a=\lim _{\epsilon_{\mathrm{e}} \rightarrow \infty} \varphi\left(\epsilon_{\mathrm{e}}\right)
$$

determines the rate of sliding in junctions for a developed plastic flow.

At small strains, a chain is modelled as a linear elastic solid with the mechanical energy

$$
w=\frac{1}{2} \mu \epsilon_{\mathrm{e}}^{2}
$$


where $\mu$ is an average rigidity per chain. Multiplying the energy, $w$, by the number of chains per unit volume, $n_{0}$, we find the strain energy density per unit volume of a nanocomposite

$$
W=\frac{1}{2} E \epsilon_{\mathrm{e}}^{2}
$$

where $E=\mu n_{0}$ is an elastic modulus.

It is worth noting that $E$ may differ from Young's modulus that is conventionally determined as the tangent of the angle between the tangent straightline to a stressstrain curve at small strains and the horizontal axis. These two quantities coincide when the elastic strain, $\epsilon_{\mathrm{e}}$, equals the macro-strain, $\epsilon$. When the stress-strain diagram has a pronounced curvature, $E$ exceeds Young's modulus found by the traditional method.

For isothermal uniaxial deformation, the Clausius-Duhem inequality reads

$$
Q(t)=-\frac{d W}{d t}(t)+\sigma(t) \frac{d \epsilon}{d t}(t) \geq 0
$$

where $Q$ is internal dissipation per unit volume. Substition of Eqs. (28) to (30) into this equality results in

$$
Q(t)=\left[\sigma(t)-E \epsilon_{\mathrm{e}}(t)\left(1-\varphi\left(\epsilon_{\mathrm{e}}(t)\right)\right)\right] \frac{d \epsilon}{d t}(t) \geq 0
$$

Assuming the expression in square brackets to vanish, we arrive at the stress-strain relation

$$
\sigma(t)=E \epsilon_{\mathrm{e}}(t)\left[1-\varphi\left(\epsilon_{\mathrm{e}}(t)\right)\right] .
$$

To approximate experimental data, we use the phenomenological equation

$$
\varphi\left(\epsilon_{\mathrm{e}}\right)=a\left[1-\exp \left(-\frac{\epsilon_{\mathrm{e}}}{\varepsilon}\right)\right]
$$

which is determined by two adjustable parameters, $a$ and $\varepsilon>0$.

Constitutive equations (28), (29), (31) and (32) contain 3 adjustable parameters:

1. the elastic modulus $E$,

2. the rate of developed plastic flow $a$,

3. the strain, $\varepsilon$, that characterizes transition to a steady plastic flow.

These quantities are found by matching the experimental data depicted in Figures 6 to 10 .

\section{$6 \quad$ Fitting observations in mechanical tests}

It follows from Eqs. (28), (29), (31) and (32) that in a uniaxial tensile test, the longitudinal stress, $\sigma$, is given by

$$
\sigma(\epsilon)=E\left(\epsilon-\epsilon_{\mathrm{p}}\right)\left\{1-a\left[1-\exp \left(-\frac{\epsilon-\epsilon_{\mathrm{p}}}{\varepsilon}\right)\right]\right\},
$$


where the plastic strain, $\epsilon_{\mathrm{p}}$, satisfies the nonlinear differential equation

$$
\frac{d \epsilon_{\mathrm{p}}}{d \epsilon}(\epsilon)=a\left[1-\exp \left(-\frac{\epsilon-\epsilon_{\mathrm{p}}}{\varepsilon}\right)\right], \quad \epsilon_{\mathrm{p}}(0)=0 .
$$

We begin with matching the experimental data in a test on the neat vinyl ester resin. To find the constants, $E, a$ and $\varepsilon$, we fix some intervals $\left[0, a_{\max }\right]$ and $\left[0, \varepsilon_{\max }\right]$, where the "best-fit" parameters $a$ and $\varepsilon$ are assumed to be located, and divide these intervals into $I$ subintervals by the points $a_{i}=i \Delta a$ and $\varepsilon_{j}=j \Delta \varepsilon(i, j=1, \ldots, I)$ with $\Delta a=a_{\max } / I$ and $\Delta \varepsilon=\varepsilon_{\max } / I$. For any pair, $\left\{a_{i}, \varepsilon_{j}\right\}$, Eq. (34) is integrated numerically by the Runge-Kutta method with the step $\Delta \epsilon=1.0 \cdot 10^{-5}$. Given $\left\{a_{i}, \varepsilon_{j}\right\}$, the elastic modulus $E=E(i, j)$ is found by the least-squares technique from the condition of minimum of the function

$$
F=\sum_{\epsilon_{k}}\left[\sigma_{\exp }\left(\epsilon_{k}\right)-\sigma_{\text {num }}\left(\epsilon_{k}\right)\right]^{2},
$$

where the sum is calculated over all experimental points, $\epsilon_{k}$, depicted in Figure 6 , $\sigma_{\exp }$ is the stress measured in a tensile test, and $\sigma_{\text {num }}$ is given by Eq. (33). The "best-fit" parameters $a$ and $\varepsilon$ minimize the function $F$ on the set $\left\{a_{i}, \varepsilon_{j} \quad(i, j=1, \ldots, I)\right\}$. Fitting the observations results in $\varepsilon=2.11 \cdot 10^{-3}$.

We fix this value of $\varepsilon$ and proceed with matching observations in tensile tests on other specimens by using only two material constants, $E$ and $a$, which are determined by the above algorithm. Figures 6 to 10 demonstrate fair agreement between the observations and the results of numerical simulation.

The average values of $E$ and $a$ (over 4 samples for any concentration of clay) are depicted in Figures 16 and 17, where the vertical bars stand for the standard deviations. Figure 16 shows that $E$ is practically independent of the clay content.

The effect of clay content, $\nu$, on the rate of developed plastic flow, $a$, is described by the phenomenological equation

$$
a=a_{0}+a_{1} \exp \left(-\frac{\nu}{\nu_{0}}\right)
$$

where $\nu_{0}$ is found by matching experimental data for the coefficient of diffusion, $D$, and the coefficions, $a_{0}$ and $a_{1}$ are determined by the least-squares method. Figure 17 demonstrates good agreement between the observations and their approximation by Eq. (35).

\section{Discussion}

Changes in the elastic modulus of a hybrid nanocomposite may be explained by the influence of two factors. On the one hand, it is conventionally accepted that reinforcement of a polymeric matrix by rigid particles results in an increase in the elastic modulus of the composite. On the other hand, clay platelets in a melt-intercalated nanocomposite screen the macro-strain, which is not transmitted to chains in the close vicinity of nanoparticles (in particular, to chains intercalated into the galleries between silica sheets). As a result, meso-regions of "occluded" polymer arise similar to the domains of occluded rubber in particle-reinforced elastomers [31]. These occluded regions are not deformed, which implies that the total mechanical energy of a nanocomposite (per unit volume) and, as a 
consequence, its elastic modulus, $E$, decrease with $\nu$. Our observation that the elastic modulus, $E$, is independent of the clay content, $\nu$, (Figure 16) may be ascribed to a cumulative effect of these two micro-mechanisms.

Weak changes in the elastic modulus of intercalated nanocomposites and even its decrease with clay content were observed by several authors. Bharadwaj et al. [8] and Xu et al. 32 reported a decrease in the elastic modulus of nanocomposites with polyester and epoxy resin matrices, respectively, and associated this behavior with a strong decay in the degree of cross-linking induced by the presence of clay particles [8]. Lepoittevin et al. [33] observed a decrease in Young's modulus for poly( $\varepsilon$-caprolactone) filled with a non-modified MMT clay and a propounced growth of the elastic modulus of the nanocomposites with organo-modified clay particles, but did not provide explanations for their findings. Kim et al. [34] suggested that changes in elastic moduli and toughness of nanocomposites may be attributed to nucleation of micro-voids in the vicinity of stacks of silicate sheets that are thought of as initiation sites for cavitation. This indicates that physical mechanisms governing the effect of clay concentration on elastic moduli of hyrid nanocomposites remain a subject of debate.

Figure 17 demonstrates that the rate of developed plastic flow, $a$, monotonically decreases with the clay content, $\nu$. This trend is in agreement with a conventional standpoint [35] that the presence of nanoparticles in an intercalated nanocomposite leads to reduction of segmental mobility of polymeric chains confined to galleries between clay platelets. It is also confirmed by DSC measurements 29] that show a pronounced increase in the glass transition temperature, $T_{\mathrm{g}}$, with the clay content (the growth of $T_{\mathrm{g}}$ confirms a decrease in molecular mobility of the host matrix).

Figure 11 reveals a similar decrease in the rate of transport of moisture molecules through the nanocomposite. Fair approximation of the experimental data depicted in Figures 11 and 17 by the exponential functions with a fixed constant, $\nu_{0}$, implies that the same micro-mechanism may be responsible for the effect of clay content on the quantities $D$ and $a$. According to the free volume concept [36, 37], an elementary mass-transport event is rationalized as follows. At some instant, $t$, a small penetrant molecule in a host polymer is at rest (thermal oscillations are disregarded) waiting for creation of a micro-hole nearby. When a hole with a sufficient size is formed (due to thermal motion of chains), the penetrant molecule hops into the hole, and remains in the new position until a new hole arises in its neighborhood. Although this picture is oversimplified (such important factors are neglected as clustering of water molecules, their weak bonding to polymeric chains, obstacles on the path of a penetrant molecule caused by the presence of inclusions), it provides a clear indication that mass transport is strongly influenced by mobility of chains. Resemblance of the graphs presented in Figures 11 and 17 implies that moisture diffusion and elastoplasticity of a nanocomposite may reflect the same phenomena at the micro-level associated with molecular mobility of the polymeric matrix.

According to the above scenario of the mass-transport process as a sequence of hopping and waiting events, slowing down of water diffusion (observed as a pronounced decrease in diffusivity $D$ ) implies that the waiting times strongly increase. As a consequence, the probability of aggregation of penetrant molecules grows, which results in formation of small clusters of water in a nanocomposite. Clustering of small molecules in solid polymers was observed in several studies, see [38, 39, 40] and the references therein. 
According to the free volume theory, clustering of water molecules implies an increase in the total mass uptake by the polymeric matrix (because penetrant molecules weakly bounded into aggregates occupy less volume than individual molecules). This conclusion is confirmed by the experimental data for $R_{\text {pol }}$ plotted in Figure 14. It follows from phenomenological equations (22) and (26) that an increase in the moisture uptake with clay content strongly correlates with a decrease in diffusivity (the same parameter, $\nu_{0}$, is used to fit the observations depicted in Figures 11 and 14).

The coefficient of diffusion, $D$, may serve as an average (over a macro-volume) rate of molecular transport. The local rate of moisture diffusion can substantially differ in the close vicinities of the clay particles (where molecular mobility is severely reduced due to intercalation of chains into galleries between platelets) and in the bulk (where mobility of chains is weakly affected by the presence of filler). This implies that the mass transport in a nanocomposite becomes substantially heterogeneous.

According to the concept of water clustering, noticeable slowing down of diffusion near inclusions implies that clusters of penetrant molecules are mainly formed in neighborhoods of clay particles. This means that immobilization of a water molecule on the hydrophilic surface of a clay layer may occur in two different ways:

1. as an one-step event, when an individual molecule merges with an unoccupied site of the surface of a silicate sheet,

2. as a two-step event, when a water molecule (i) separates from a cluster, and (ii) merges with an appropriate site.

At small concentrations of clay, when diffusivity, $D$, is rather large and clustering of water molecules is negligible, immobilization of small molecules takes place by the one-step mechanism, which means that the rate of bounding of penetrant molecules is relatively high. With an increase in the clay content (which is tantamount to a decrease in the average diffusivity, $D$, see Figure 11), the one-step mechanism of bounding is replaced by the two-step mechanism. According to the proposed scenario, these changes in the immobilization mechanism result in a noticeable deceleration of the process of water bounding to silica sheets, because separation of a water molecule from a cluster requires an additional energy provided by thermal fluctuations. This slowing down (reflected by the model as a decrease in the coefficient $K$ ) is confirmed by the experimental data presented in Figure 13. This figure demonstrates that $K$ is reduced by at least two orders of magnitude with the growth of clay content.

It is natural to assume that the total moisture uptake by the clay particles, $R_{\text {clay }}$, is proportional to their concentration, $\nu$. The parameter $R_{\text {clay }}$ is plotted in Figure 15 versus clay content. The experimental data show that the linear dependence (27) is in good agreement (within the experimental uncertainties) with the observations. This conclusion may be treated as a confirmation of our kinetic equations for anomalous moisture diffusion.

Another confirmation of the model is provided by the fact that the diffusivity, $D$, becomes practically independent of the clay content when $\nu$ exceeds 5 wt.-\% (Figure 11). This conclusion is in accord with numerous observations that reveal substantial improvement of material properties of hybrid nanocomposites with clay content in the range of several (conventionally, 5) wt.- $\%$, whereas at higher clay fractions, material parameters are weakly affected by the MMT concentration. 


\section{Concluding remarks}

Diffusion tests and uniaxial tensile tests with a constant strain rate have been performed on an intercalated nanocomposite with vinyl ester resin matrix and montmorillonite clay filler at room temperature. Observations in diffusion tests show that moisture diffusion in the neat resin is Fickian, whereas it becomes noticeably anomalous (non-Fickian) with the growth of the clay content. This transition is attributed to immobilization of the penetrant molecules on hydrophilic surfaces of clay layers. Experimental data in mechanical tests demonstrate that the response of the neat vinyl ester resin is strongly elastoplastic, whereas plastic strains strongly decrease with the clay content. This observations are ascribed to slowing down of molecular mobility in the host matrix driven by confinement of chains in galleries between clay layers.

Constitutive equations have been developed for anomalous moisture diffusion through and for the elastoplastic behavior of a nanocomposite. These relations are determined by 3 adjustable parameters (for each process) that are found by fitting the experimental data. Fair agreement is demonstrated between the observations and the results of numerical simulation.

The following conclusions are drawn:

1. The diffusivity, $D$, and the rate of developed plastic flow, $a$, decrease with clay content following similar dependences. The effect of clay concentration, $\nu$, on these quantities may be explained by slowing down of molecular mobility in the polymeric matrix.

2. The moisture uptake by the host matrix grows with clay content. With reference to the free volume concept, this observation is explained by clustering of water molecules in the close vicinity of stacks of platelets (where diffusivity falls down dramatically).

3. The rate of immobilization of water molecules on the hydrophilic surfaces of inorganic sheets decreases noticeably with $\nu$, which is associated with transition from the one-step to the two-step mechanism of immobilization.

An important advantage of our model compared to the tortuous path concept is that it can explain (in a unified manner) a decay in diffusivity of a hybrid nanocomposite (with the clay content) together with an increase in the maximal moisture uptake by the polymeric matrix and a reduction of its elastoplastic properties.

It is worth noting that the correlations revealed between a decrease in diffusivity and a decrease in the rate of a steady plastic flow provide a way to substantially reduce the number of (time-consuming) diffusion tests by replacing a part of experiments by mechanical tests that do not require long-time observations. 


\section{References}

[1] Yano, K.; Usuki, A.; Okada, A.; Kurauchi, T.; Kamigaito, O. J Polym Sci Polym Chem 1993, 31, 2493-2498.

[2] Lan, T.; Padmananda, D.K.; Pinnavaia, T.J. Chem Mater 1994, 6, 573.

[3] Burnside, S.D.; Giannelis, E.P. Chem Mater 1995, 7, 1597.

[4] Messersmith, P.B.; Giannelis, E.P. J Polym Sci Polym Chem 1995, 33, 1047-1057.

[5] Yano, K.; Usuki, A.; Okada, A. J Polym Sci Polym Chem 1997, 35, 2289-2294.

[6] Petricova, E.; Knight, R.; Schadler, L.S.; Twardowski, T.E. J Appl Polym Sci 2000, 78, 2272-2289.

[7] Beall, G.W. In: Pinnavaia, T.J., Beall, G.W. (eds.) Polymer-Clay Nanocomposites. Wiley, New York, 2001, pp. 267-279.

[8] Bharadwaj, R.K.; Mehrabi, A.R.; Hamilton, C.; Trujilo, C.; Murga, M.; Fan, R.; Chavira, A.; Thompson, A.K. Polymer 2002, 43, 3699-3705.

[9] Gorpasi, G.; Tortora, M.; Vittoria, V.; Galli, G.; Chiellini, E. J Appl Polym Sci Polym Phys 2002, 40, 1118-1124.

[10] Tong X.; Zhao, H.; Tang, T.; Feng, Z.; Huang, B. J Polym Sci Polym Chem 2002, 40, 1706-1711.

[11] Tortora, M.; Vittoria, V.; Galli, G.; Ritrovati, S.; Chielline, E. Macromol Mater Eng 2002, 287, 243-249.

[12] Wu, Z.; Zhou, C.; Qi, R.; Zhang, H. J Appl Polym Sci 2002, 83, 2403-2410.

[13] Nielsen, L.E. J Macromol Sci Chem 1967, A1, 929.

[14] Cussler, E.L.; Hughes, S.E.; Ward, W.J.; Aris, R. J Membr Sci 1988, 38, 161.

[15] Fukuda, M.; Kuwajima, S. J Chem Phys 1997, 107, 2149-2159.

[16] Fredrickson, G.H.; Bicerano, J. J Chem Phys 1999, 110, 2181-2188.

[17] Bharadwaj, R.K. Macromolecules 2001, 34, 9189-9192.

[18] Colombini, D.; Martinez-Vega, J.J.; Merle, G. Polymer 2002, 43, 4479-4485.

[19] Gloaguen, J.M.; Lefebvre, J.M. Polymer 2001, 42, 5841-5847.

[20] Patel, S.V.; Raval, D.K.; Thakkar, J.R. Angew Makromol Chem 1999, 265, 13-15.

[21] Cai, L.W.; Weitsman, Y. J Compos Mater 1994, 28, 130-154. 
[22] Vanlandingham, M.R.; Eduljee, R.F.; Gillespie, J.W. J Appl Polym Sci 1999, 71, 787-798.

[23] Roy, S.; Xu, W.X.; Park, S.J.; Liechti, K.M. Trans ASME J Appl Mech 2000, 67, 391-396.

[24] Chen, C.; Han, B.; Li, J.; Shang, T.; Zou, J.; Jiang, W. J Membr Sci 2001, 187, 109-118.

[25] Uschitsky, M.; Suhir, E. Trans ASME J Electronic Packaging 2001, 123, 47-51.

[26] Carter, H.G.; Kibler, K.G. J Compos Mater 1978, 12, 118-130.

[27] Gurtin, M.E.; Yatomi, C. J Compos Mater 1979, 13, 126-130.

[28] Drozdov, A.D.; Christiansen, J. deC.; Gupta, R.K.; Shah, A.P. E-preprint condmat/0205469.

[29] Shah, A.P.; Gupta, R.K.; GangaRao, H.V.S.; Powell, C.E. Polym Eng Sci 2002, accepted.

[30] Messersmith, P.B.; Giannelis, E.P. Chem Mater 1994, 6, 1719-1725.

[31] Witten, T.A.; Rubinstein, M.; Colby, R.H. J Phys II (France) 1993, 3, 367.

[32] Xu, W.-B.; Bao, S.-P.; He, P.-S. J Appl Polym Sci 2002, 84, 842-849.

[33] Lepoittevin, B.; Devalckenaere, M.; Pantoustier, N.; Alexandre, M.; Kubies, D.; Jerome, R.; Dubois, P. Polymer 2002, 43, 4017-4023.

[34] Kim, G.-M.; Lee, D.-H.; Hoffmann, B.; Kressler, J.; Stöppelmann, G. Polymer 2001, 42, 1095-1100.

[35] Hwu, J.M.; Jiang, G.J.; Gao, Z.M.; Xie, W.; Pan, W.P. J Appl Polym Sci 2002, 83, 1702-1710.

[36] Cohen, M.H.; Turnbull, D. J Chem Phys 1959, 31, 1164.

[37] Litt, M. J Rheol 1986, 30, 853-868.

[38] Xu, G.; Gryte, C.C.; Nowick, A.S.; Li, S.Z.; Pak, Y.S.; Greenbaum, S.G. J Appl Phys 1989, 66, 5290-5296.

[39] Stapf, S.; Kimmich, R.; Seitter, R.-O. Phys Rev Lett 1995, 75, 2855-2858.

[40] Sammon, C.; Mura, C.; Yarwood, J.; Everall, N.; Swart, R.; Hodge, D. J Phys Chem B 1998, 102, 3402-3411. 


\section{List of figures}

Figure 1: The relative water uptake $\Phi$ versus the reduced time $\bar{t}\left(\mathrm{~h}^{\frac{1}{2}} / \mathrm{mm}\right)$. Circles: experimental data $(2 l=0.49953 \mathrm{~mm}, \nu=0.0$ wt.-\%). Solid line: results of numerical simulation

Figure 2: The relative water uptake $\Phi$ versus the reduced time $\bar{t}\left(\mathrm{~h}^{\frac{1}{2}} / \mathrm{mm}\right)$. Circles: experimental data $(2 l=0.17318 \mathrm{~mm}, \nu=0.5 \mathrm{wt} .-\%)$. Solid line: results of numerical simulation

Figure 3: The relative water uptake $\Phi$ versus the reduced time $\bar{t}\left(\mathrm{~h}^{\frac{1}{2}} / \mathrm{mm}\right)$. Circles: experimental data $(2 l=0.18344 \mathrm{~mm}, \nu=1.0 \mathrm{wt} . \%)$. Solid line: results of numerical simulation

Figure 4: The relative water uptake $\Phi$ versus the reduced time $\bar{t}\left(\mathrm{~h}^{\frac{1}{2}} / \mathrm{mm}\right)$. Circles: experimental data $(2 l=0.1397 \mathrm{~mm}, \nu=2.5$ wt.- $\%)$. Solid line: results of numerical simulation

Figure 5: The relative water uptake $\Phi$ versus the reduced time $\bar{t}\left(\mathrm{~h}^{\frac{1}{2}} / \mathrm{mm}\right)$. Circles: experimental data $(2 l=0.18452 \mathrm{~mm}, \nu=5.0 \mathrm{wt} . \%)$. Solid line: results of numerical simulation

Figure 6: The stress $\sigma$ MPa versus strain $\epsilon$. Circles: experimental data $(\nu=0.0$ wt.- $\%)$. Solid line: results of numerical simulation

Figure 7: The stress $\sigma$ MPa versus strain $\epsilon$. Circles: experimental data $(\nu=0.5$ wt.- $\%)$. Solid line: results of numerical simulation

Figure 8: The stress $\sigma$ MPa versus strain $\epsilon$. Circles: experimental data $(\nu=1.0$ wt.- $\%)$. Solid line: results of numerical simulation

Figure 9: The stress $\sigma \mathrm{MPa}$ versus strain $\epsilon$. Circles: experimental data $(\nu=2.5$ wt.- $\%)$. Solid line: results of numerical simulation

Figure 10: The stress $\sigma$ MPa versus strain $\epsilon$. Circles: experimental data $(\nu=5.0$ wt.- $\%)$. Solid line: results of numerical simulation

Figure 11: Diffusivity $D \cdot 10^{-7} \mathrm{~mm}^{2} / \mathrm{s}$ versus the clay content $\nu$ wt.- $\%$. Circles: treatment of observations. Solid line: approximation of the experimental data by Eq. (22) with $D_{0}=3.53$ and $D_{1}=5.93$

Figure 12: The dimensionless parameter $C$ versus the clay content $\nu$ wt.- $\%$. Circles: treatment of observations. Solid line: approximation of the experimental data by Eq. (23) with $C_{1}=0.057$

Figure 13: The rate of adhesion $K$ versus the clay content $\nu$ wt.- $\%$. Circles: treatment of observations. Solid line: approximation of the experimental data by Eq. (24) with $K_{1}=0.29$ and $K_{1}=4.80$

Figure 14: The moisture adsorption by the polymer $R_{\text {pol }} \%$ versus the clay content $\nu$ wt.-\%. Circles: treatment of observations. Solid line: approximation of the experimental data by Eq. (26) with $R_{0}=0.90$ and $R_{1}=0.49$

Figure 15: The maximal moisture uptake by the clay particles $R_{\text {clay }}=\kappa n_{1}^{\circ} / \rho \%$ versus the clay content $\nu$ wt.- $\%$. Circles: treatment of observations. Solid line: approximation 
of the experimental data by Eq. (27) with $R_{1}=0.053$

Figure 16: The elastic modulus $E$ GPa versus the clay concentration $\nu$ wt.-\%. Circles: treatment of observations. Solid line: approximation of the experimental data by the constant $E=4.60 \mathrm{GPa}$

Figure 17: The rate of developed plastic flow $a$ versus the clay content $\nu$ wt.- $\%$. Circles: treatment of observations. Solid line: approximation of the experimental data by Eq. (35) with $a_{0}=0.106$ and $a_{1}=0.106$ 


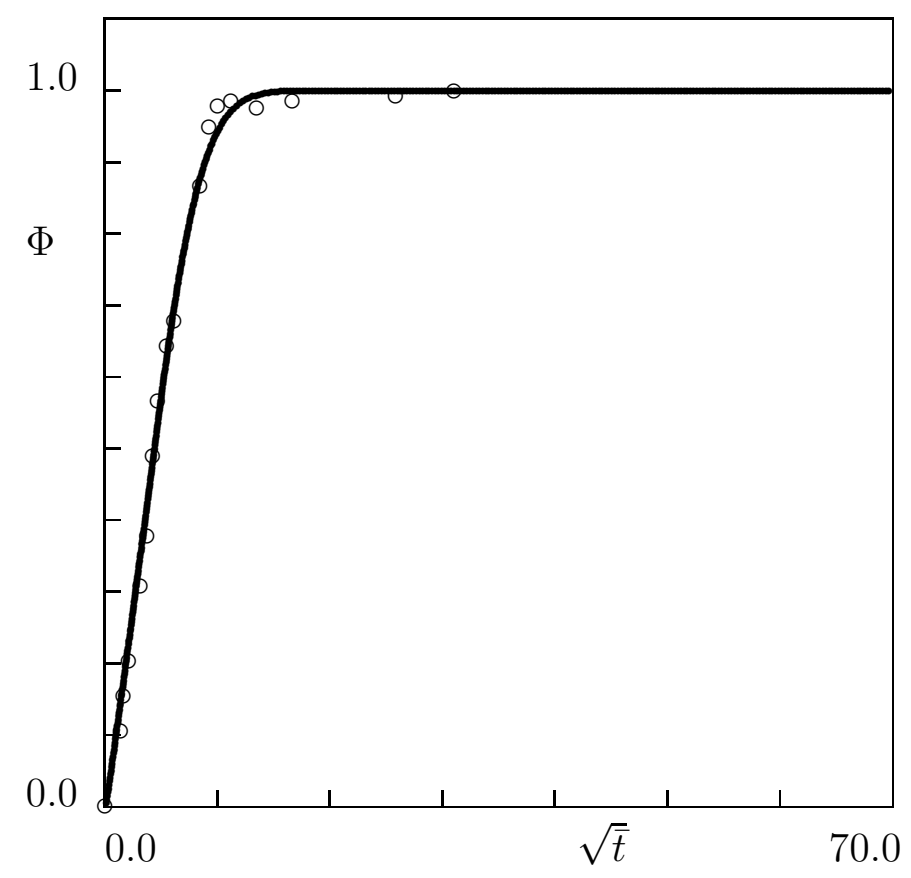

Figure 1:

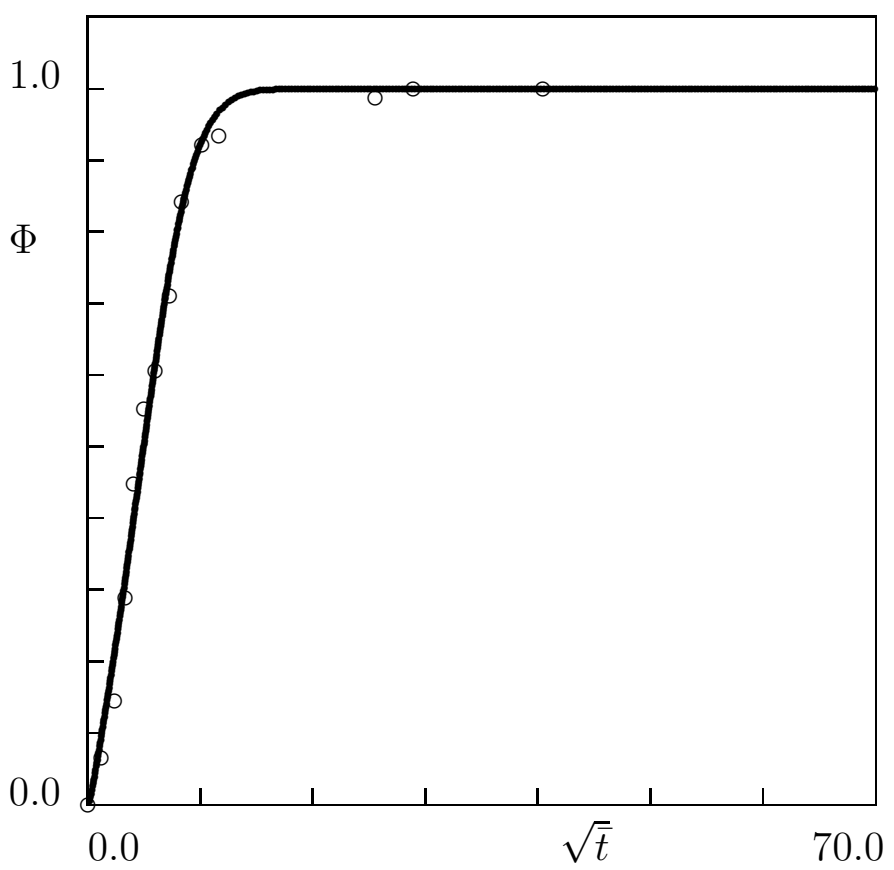

Figure 2: 


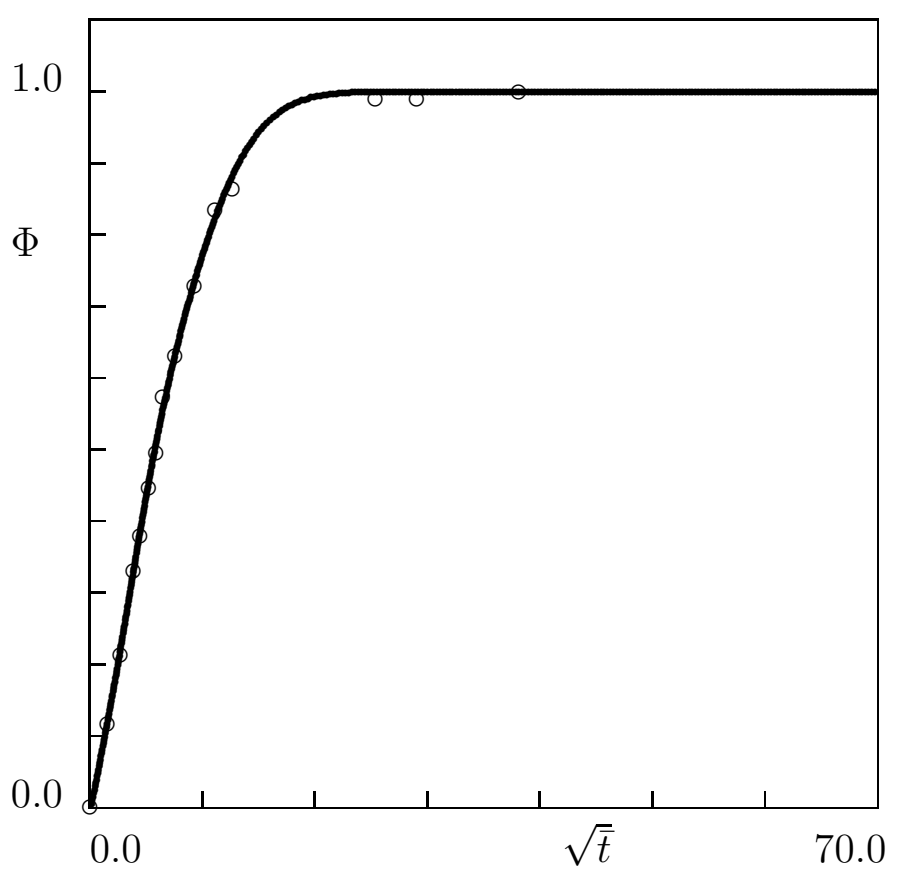

Figure 3:

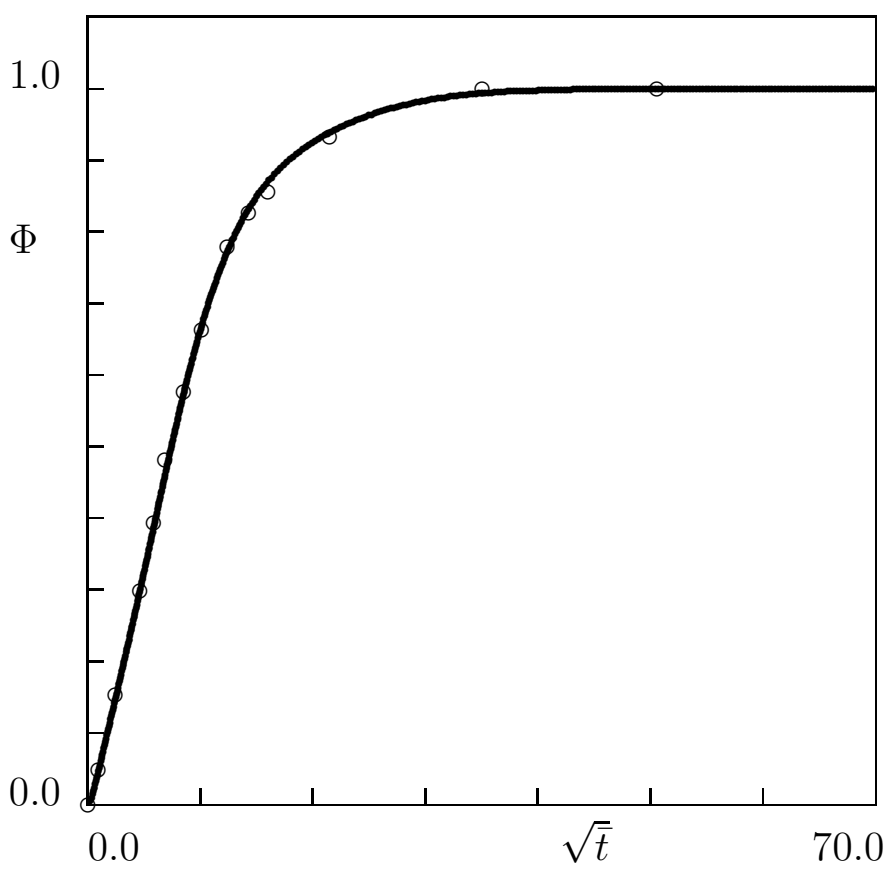

Figure 4: 


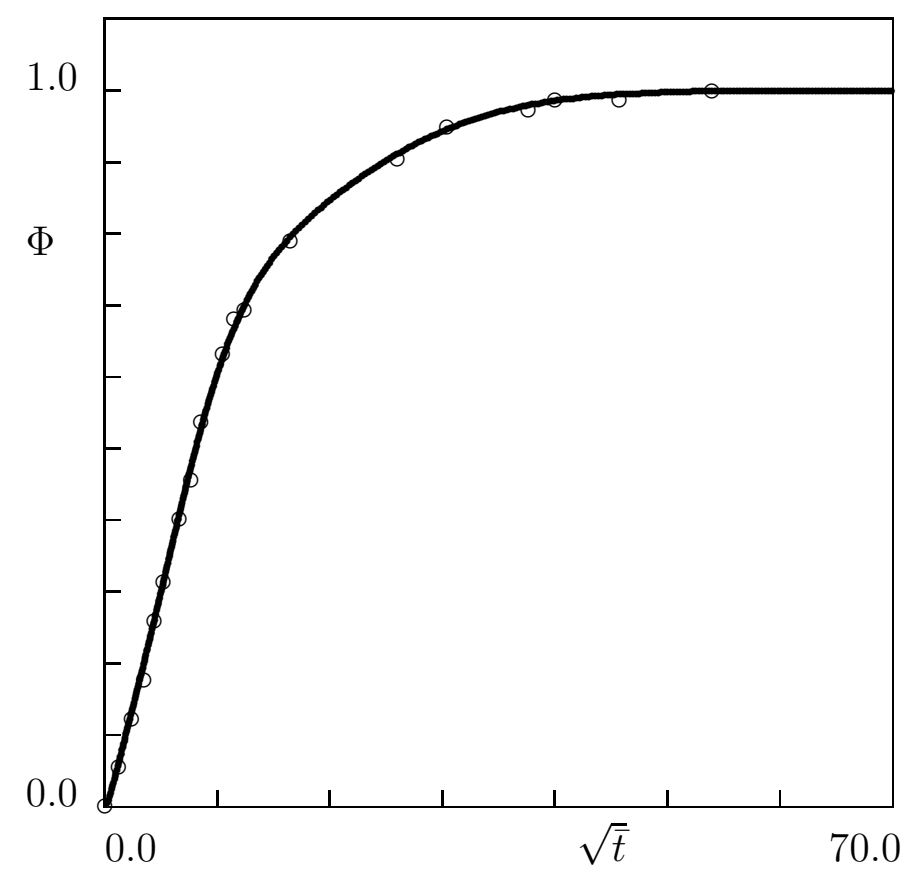

Figure 5:

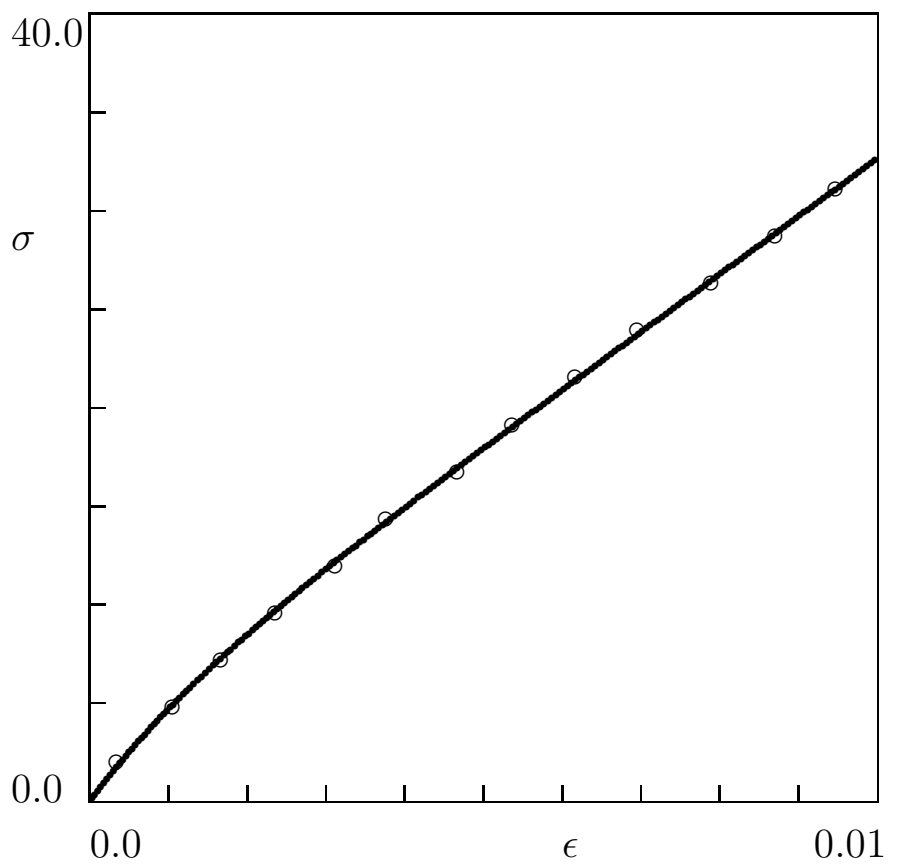

Figure 6: 


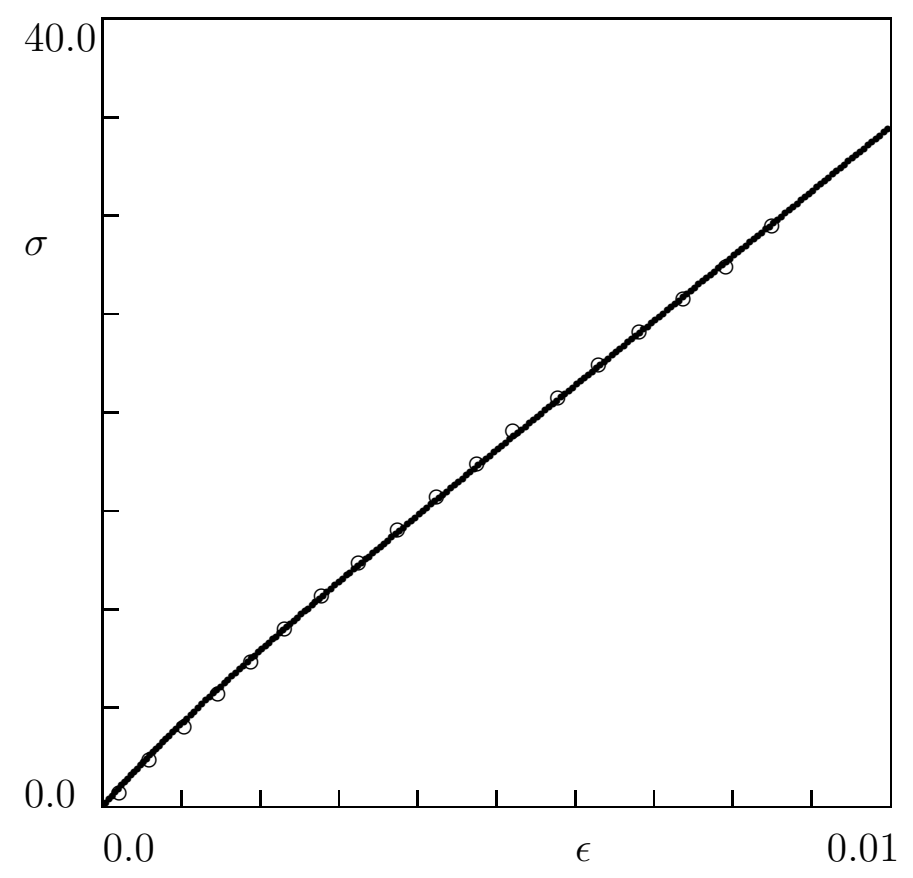

Figure 7:

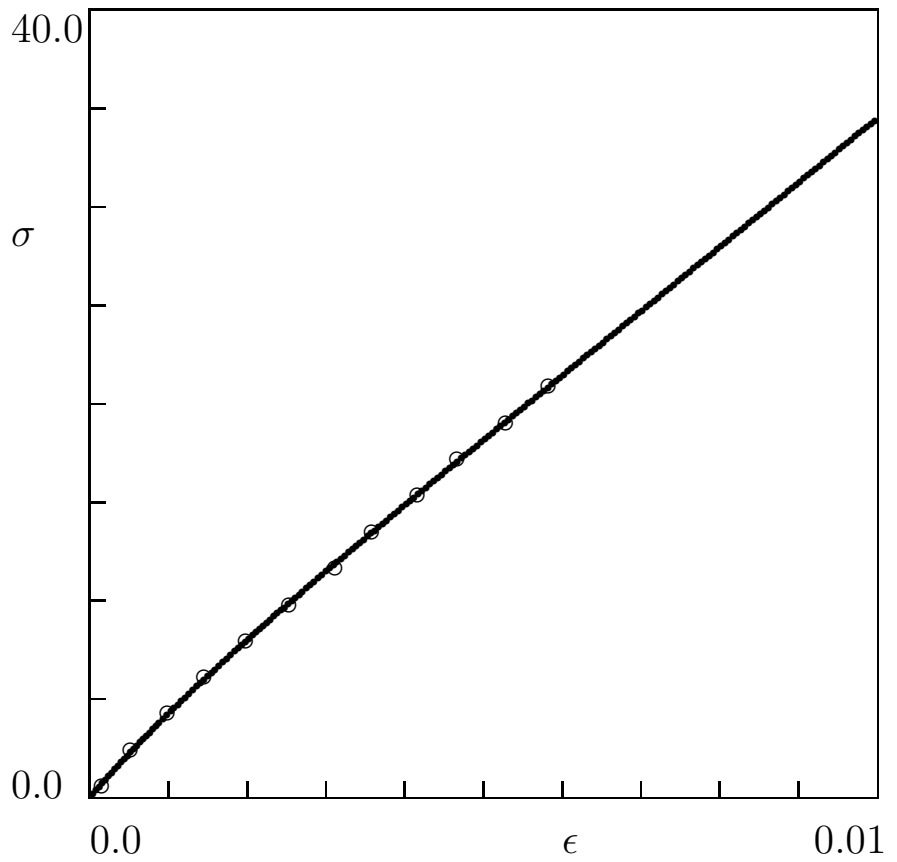

Figure 8: 


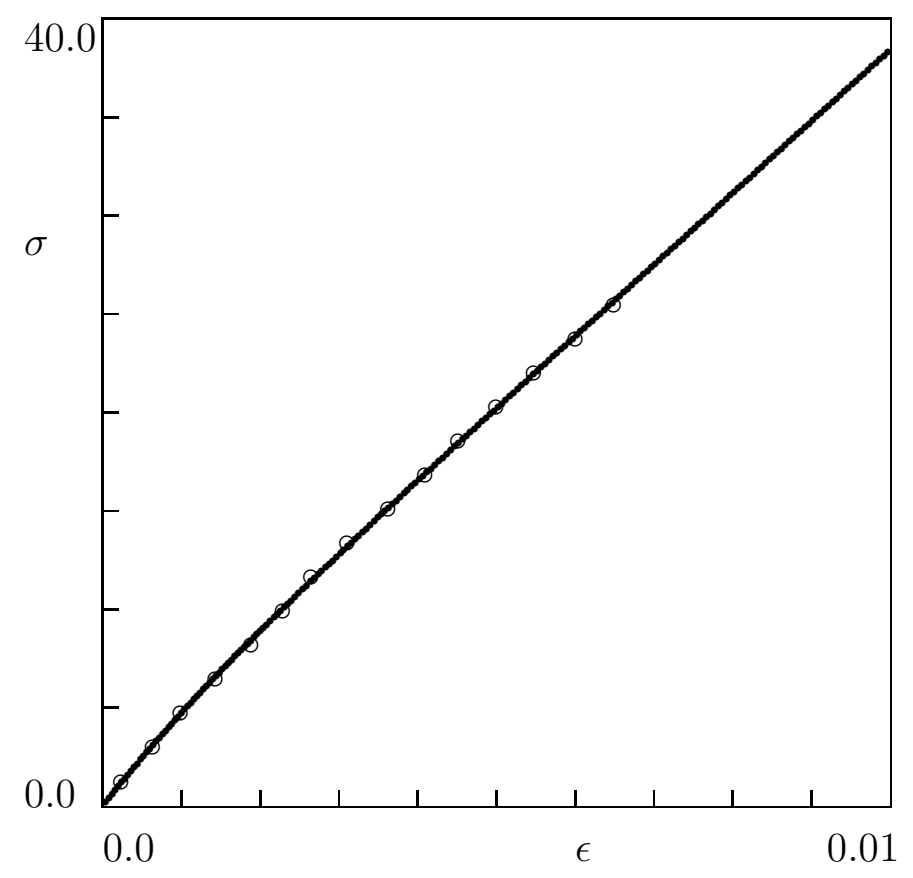

Figure 9:

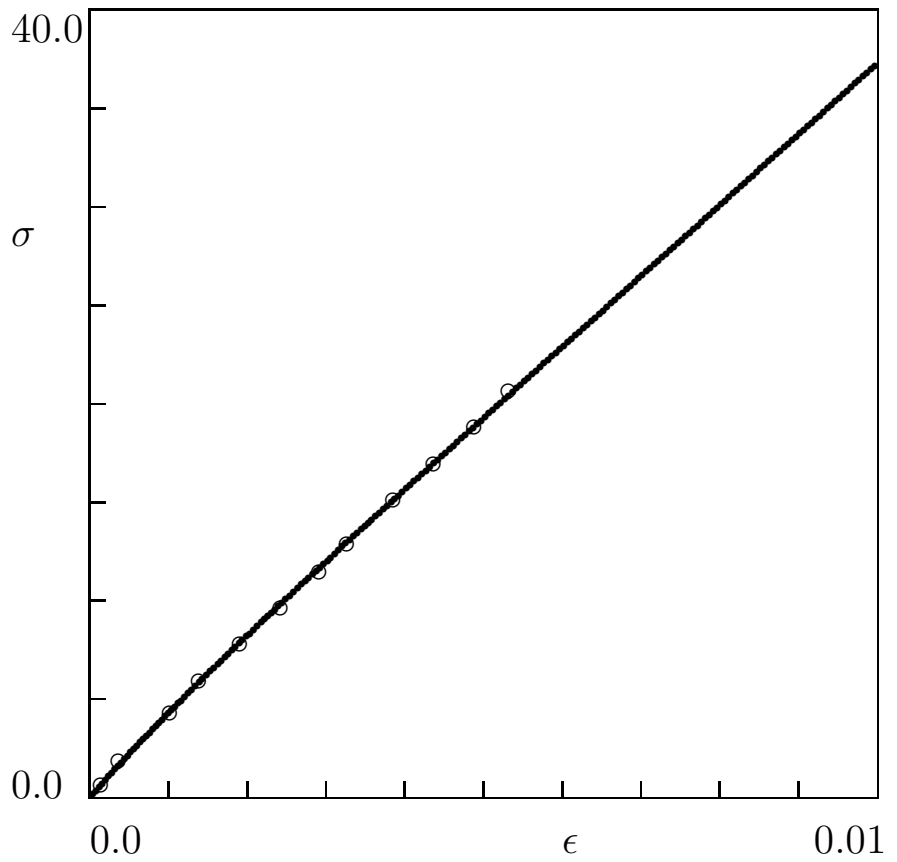

Figure 10: 


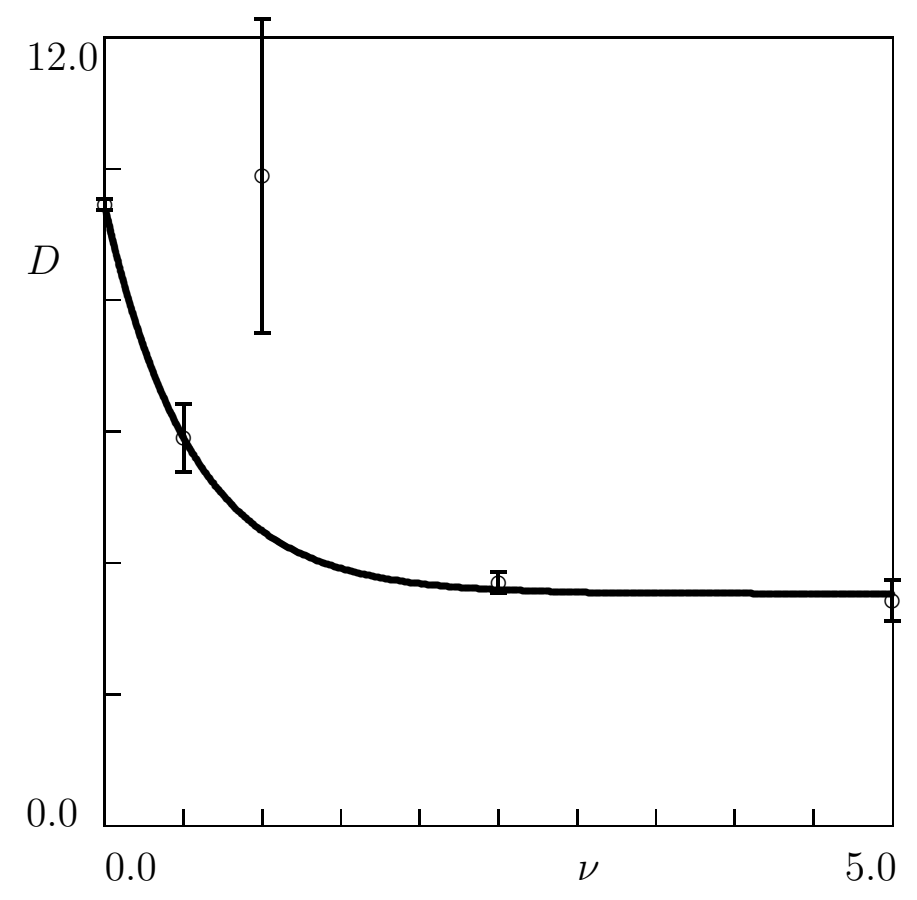

Figure 11:

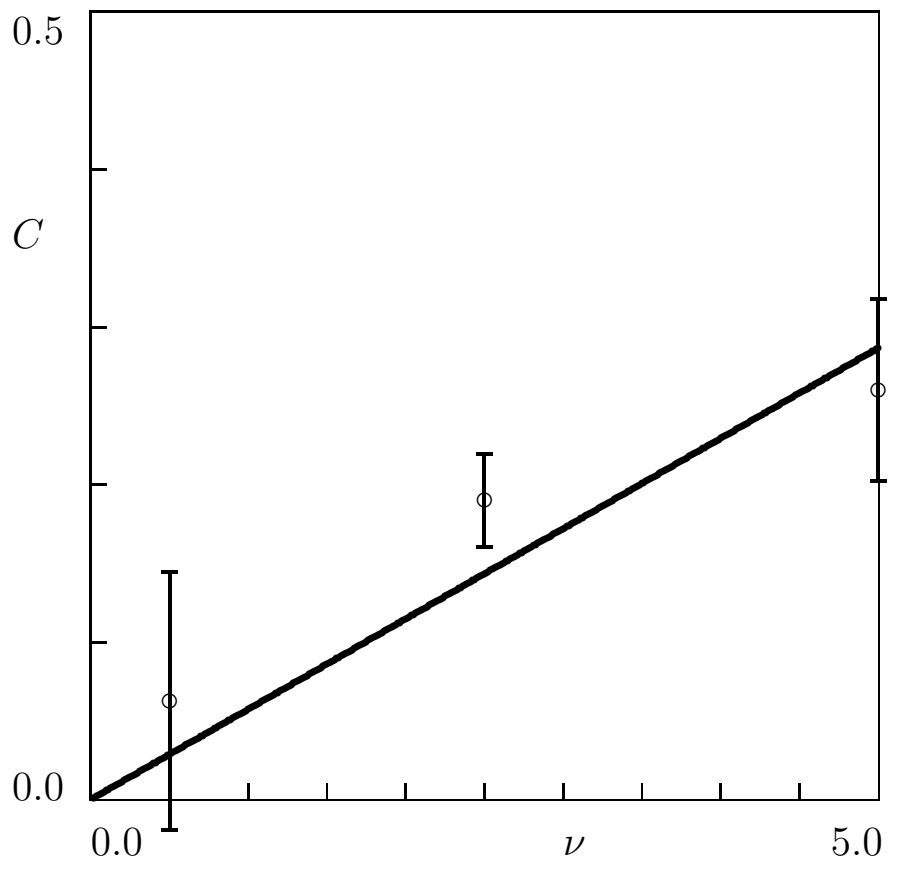

Figure 12: 


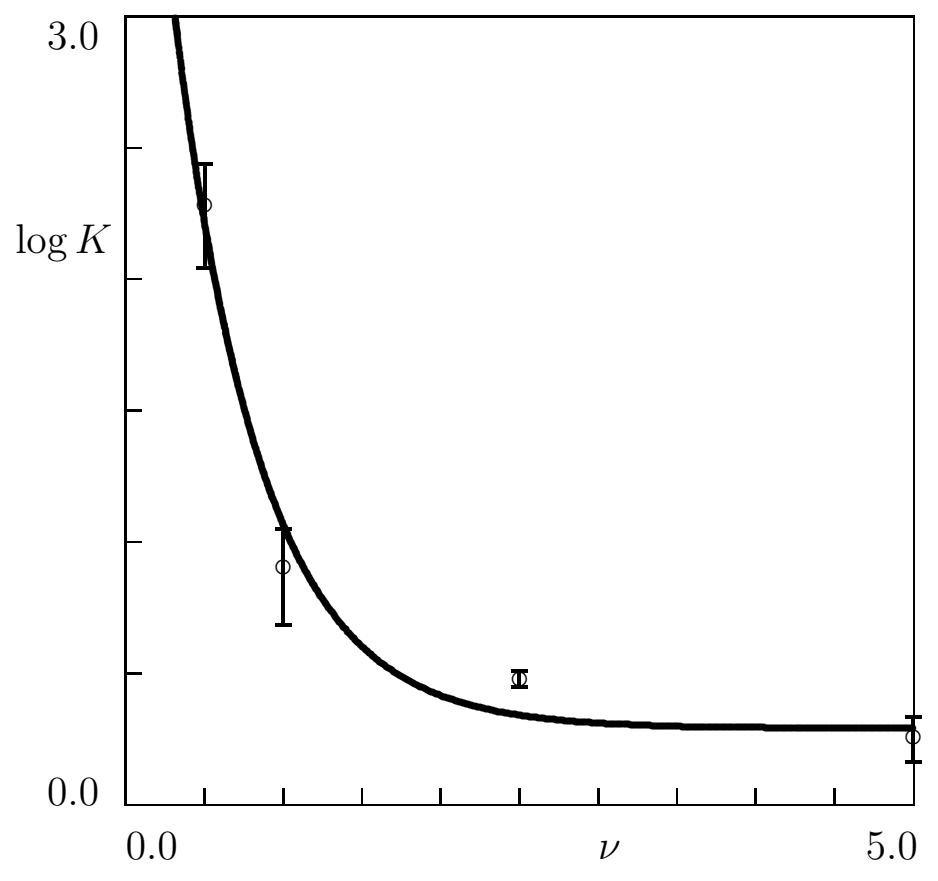

Figure 13:

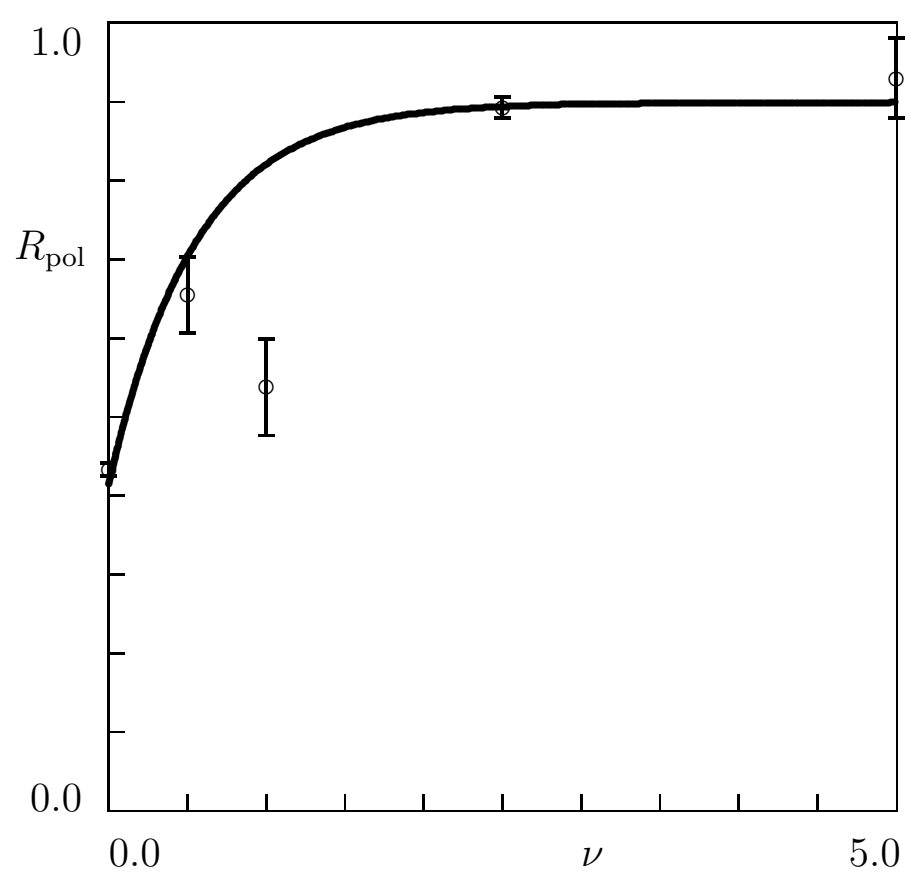

Figure 14: 


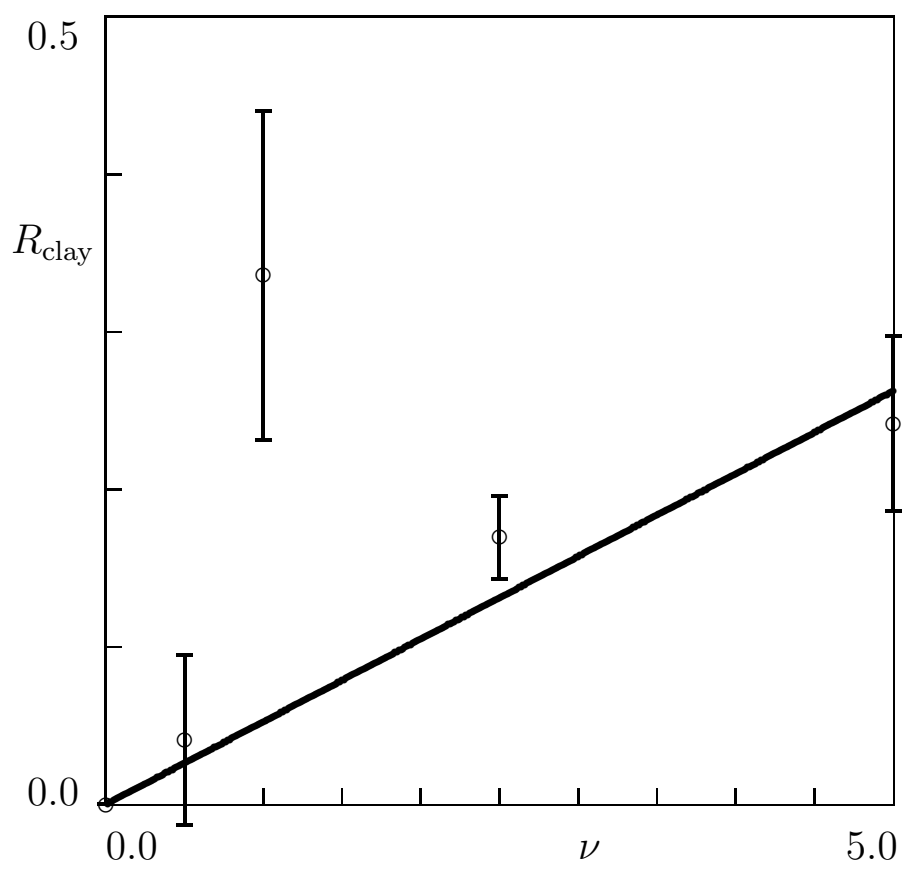

Figure 15:

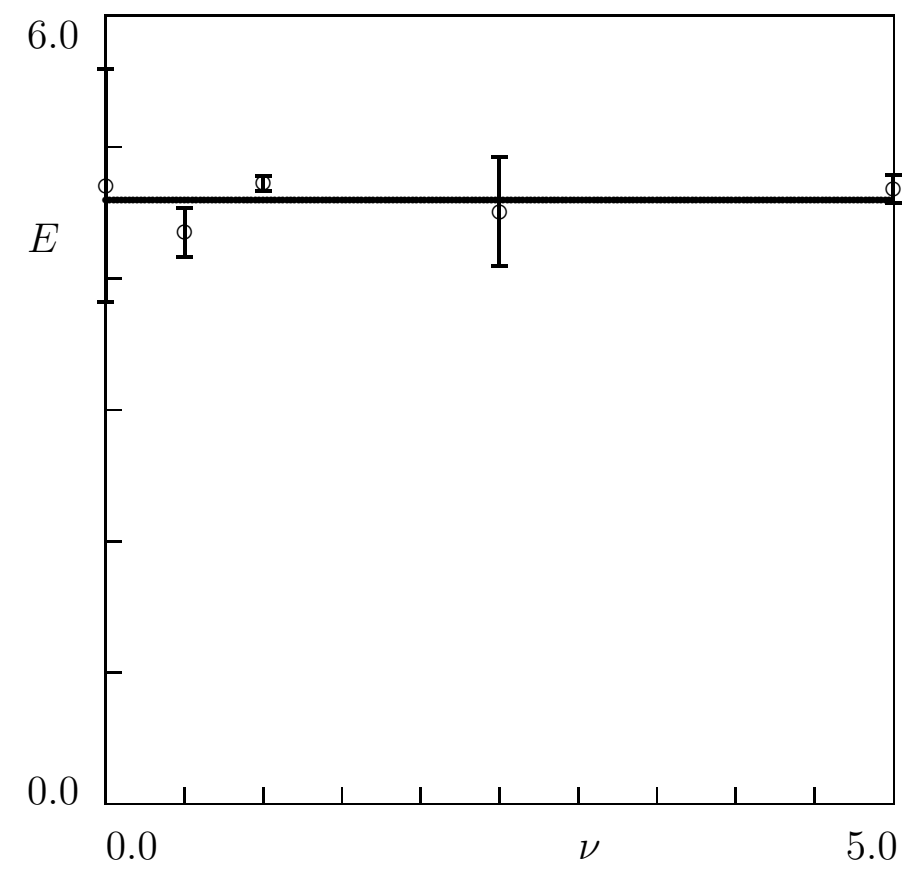

Figure 16: 


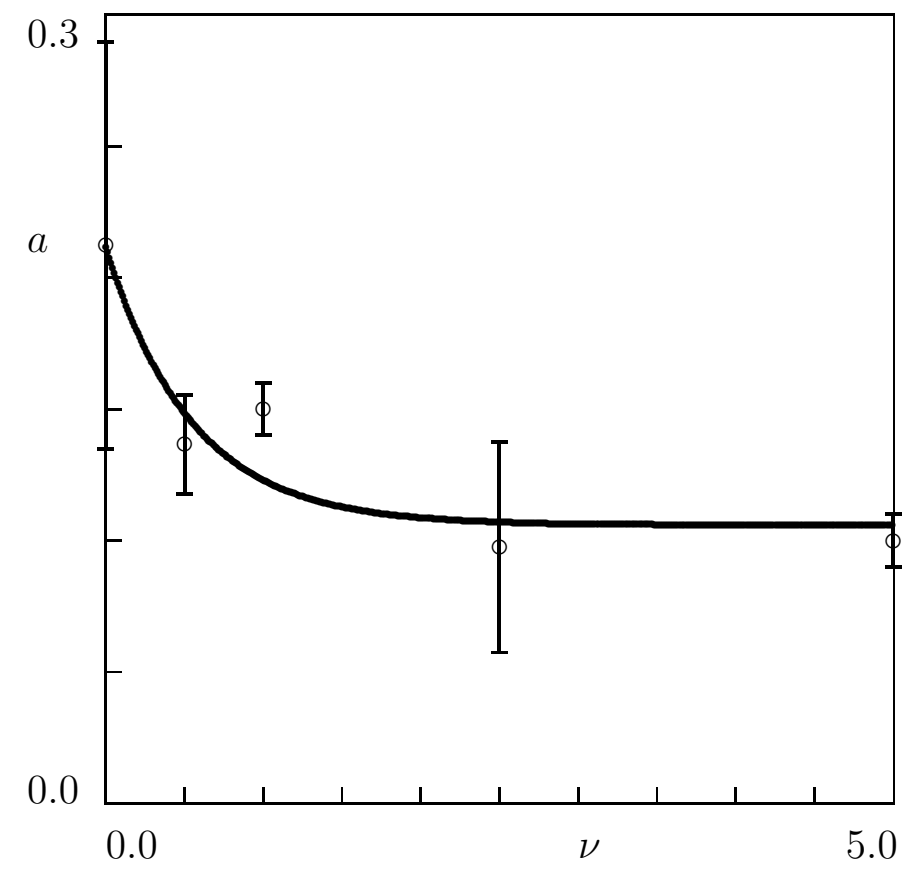

Figure 17: 